INPLASY

PROTOCOL

To cite: Yang et al. Pancreatic safety of DPP-4 Inhibitors in type 2 diabetes millitus $A$ protocol for systematic review and network meta-analysis. Inplasy protocol 202230014. doi:

10.37766/inplasy2022.3.0014

Received: 03 March 2022

Published: 03 March 2022

Corresponding author:

Fan Yang

1161410522@qq.com

Author Affiliation:

Shandong University of

Traditional Chinese Medicine.

Support: NSFC (NO.

81774254).

Review Stage at time of this submission: Piloting of the study selection process.

Conflicts of interest:

None declared.

\section{Pancreatic safety of DPP-4 Inhibitors in type 2 diabetes millitus A protocol for systematic review and network meta-analysis}

\author{
Yang, F1; Zhao, Q2; Dong, Y3; Li, B4; Ning, B5.
}

Review question / Objective: To evaluate the pancreatic safety in patients with type 2 diabetes treated with DPP-4 inhabitors as compared to placebo or other active drugs in randomized controlled trials.

Condition being studied: DPP-4i is an incretin-based pharmacotherapy, a new antidiabetic drug that differs from conventional oral glucose-lowering medications. Incretin hormones stimulate insulin secretion while inhibiting glucagon secretion in a glucose-dependent way, lowering blood glucose and reducing the risk of hypoglycemia and weight gain. In recent years, more attention has been devoted to the cardiovascular or renal outcomes of DPP-4i. However, other safety events, such as pancreatic events that are uncommon and controversial, are more likely to be overlooked. Numerous researchers have investigated this topic, and some secondary analysis has also been conducted, but no consensus has been reached based on the evidence gathered thus far. Accordingly, the safety of DPP-4i medicines on the pancreas is still a worry for diabetic individuals.

INPLASY registration number: This protocol was registered with the International Platform of Registered Systematic Review and Meta-Analysis Protocols (INPLASY) on 03 March 2022 and was last updated on 03 March 2022 (registration number INPLASY202230014).

\section{INTRODUCTION}

Review question / Objective: To evaluate the pancreatic safety in patients with type 2 diabetes treated with DPP-4 inhabitors as compared to placebo or other active drugs in randomized controlled trials.
Condition being studied: DPP-4i is an incretin-based pharmacotherapy, a new antidiabetic drug that differs from conventional oral glucose-lowering medications. Incretin hormones stimulate insulin secretion while inhibiting glucagon secretion in a glucose-dependent way, lowering blood glucose and reducing the 
risk of hypoglycemia and weight gain. In recent years, more attention has been devoted to the cardiovascular or renal outcomes of DPP-4i. However, other safety events, such as pancreatic events that are uncommon and controversial, are more likely to be overlooked. Numerous researchers have investigated this topic, and some secondary analysis has also been conducted, but no consensus has been reached based on the evidence gathered thus far. Accordingly, the safety of DPP-4i medicines on the pancreas is still a worry for diabetic individuals.

\section{METHODS}

Participant or population: Patients diagnosed with type 2 diabetes according to the criteria formulated by WHO.

Intervention: All types of DPP-4 inhibitors at clinically-relevant doses.

Comparator: Active hypoglycemic drugs or placebo.

Study designs to be included: Randomized controlled clinical trials written in English and with available data related to pancreatic safety, and in which DPP-4 inhibitors have been compared with other active drugs or placebo in patients with T2DM will be included.

Eligibility criteria: 1 . Types of participants (1) Patients diagnosed with type 2 diabetes according to the criteria formulated by WHO[7] . (2) Be over 18 years old. (3) Patients have been treated with DPP-4is or other active antidiabetic medications. (4) No restrictions on race, sex, nationality, disease severity, 2. Types of interventions and comparators We will take into account the research that evaluated the following therapies involving DPP-4is (mainly including Sitagliptin, Saxagliptin, Vildagliptin, Linagliptin, and Alogliptin) compared with placebo or other hypoglycemic drugs (like Biguanides, Sulfonylureas, Thiazolidinediones, aglycosidase inhibitors, SGLT-2 inhibitor, and DLP-1 receptor agonist) in patients with T2DM. 3. Outcomes (1) The primary outcome of our study is pancreatic safety events, such as pancreatitis and pancreatic cancer. (2) The secondary outcome of interest will include the change of pancreatic enzyme from baseline. 4. Types of studies Only randomized controlled trials (RCTs) will be enrolled in this review, which compared DPP-4i therapies with other active hypoglycemic medications or placebo in T2DM patients with a minimum intervention length of 4 weeks. Either blinding or open-label studies will be considered. Furthermore, the literature will only be available in English. Other types of studies, such as in vivo or in vitro studies, case reports, reviews, and non-RCTs, will be eliminated.

Information sources: Medline, Embase, PubMed, Web of Science, the Cochrane Central Register of Controlled Trials (CENTRAL) were searched for published trials. Clinical trial registries (such as http:// www.ClinicalTrials.gov) were also searched for unpublished trials. Moreover, we will check the systematic reviews and metaanalyses to search for further relevant studies and contact the authors to obtain incomplete data.

Main outcome(s): Pancreatic safety events involving acute pancreatitis, chronic pancreatitis or pancreatic cancer.

Additional outcome(s): The change of pancreatic enzyme from baseline.

Quality assessment / Risk of bias analysis: Two researchers will check the quality of trials involved in this meta analysis, and the discrepancies will be resolved by the third researcher. Trial quality will be assessed using the Cochrane risk of bias tool. The GRADE framework will be used to assess the quality of the evidence contributing to each network estimate.

Strategy of data synthesis: A standard pairwise meta-analysis will be performed using the random or fixed effects model. Relative Risk for pancreatic safety events will be calculated as effect measures with $95 \%$ confidence intervals. Weighted mean difference for pancreatic amylase will be 
calculated as effect measures with $95 \%$ confidence intervals. The statistic of $\mathrm{I}^{2}$ will be used to assess heterogeneity levels.

Subgroup analysis: If the results show significant heterogeneity, subgroup analyses or meta-regression analyses will be performed assessing the possible reasons and controlling the confounding factors. The analysis may be conducted according to group of age, gender, duration of disease, length of trial, baseline level of HbA1c, sample size or quality of study to assess whether the results have been impacted by these factors.

Sensitivity analysis: Several possible nodes, such as sample size, methodological weakness, and missing data, will be considered. If the heterogeneity is improved after excluding the studies with a high risk of bias, indicating that the study affects the robustness of the outcome. Oppositely, if the heterogeneity change is not significant, it indicates that the result is reliable.

Country(ies) involved: China.

Keywords: Dipeptidyl-peptidase IV inhibitors; Pancreatic safety; type 2 diabetes mellitus;Systematic review; Network meta-analysisDiabetes Mellitus, Type 2; Dipeptidyl-Peptidase IV Inhibitors; Randomized.

Contributions of each author:

Author 1 - Fan Yang.

Author 2 - Quanlin Zhao.

Author 3 - Youzi Dong.

Author 4 - Baohua Li.

Author 5 - Bobiao Ning. 